\title{
Spherical Agglomeration of Platy Crystals: Curious Case of Etodolac
}

\author{
Supriya Jitkar $\$$, Rajesh Thipparaboina $\$$, Rahul B Chavan, Nalini R Shastri*
}

Solid State Pharmaceutical Research Group (SSPRG), Department of Pharmaceutics, National Institute of Pharmaceutical Education and Research (NIPER), Hyderabad, India

${ }^{\$}$ Both the authors contributed equally

*Corresponding author. Nalini R Shastri

Tel. $+91-040-23423749$

Fax. $+91-040-23073751$

E-mail: nalini@niperhyd.ac.in,svcphod@yahoo.co.in

Address: Department of Pharmaceutics, NIPER (National Institute of Pharmaceutical Education \& Research), Balanagar, Hyderabad, India, Pin Code - 500037.

\section{Supporting information}

\section{Basic Pharmaceutical Terms used in the manuscript}

Angle of repose: Angle of repose is a characteristic term related to interparticulate friction or resistance to movement between particles. It is a measure of flow of the powder. Smaller the angle of repose better is the flow. An angle of repose of up to $25^{\circ}$ is an indicative of good flow

property. ${ }^{1,2}$ Angle of repose is not an intrinsic property of the powder; i.e., it is dependent upon the method used to form the cone of powder. The peak of the cone of powder can be distorted by the impact of powder from above. By carefully building the powder cone, the distortion caused by impact can be minimized. The nature of the base upon which the powder cone is formed influences the angle of repose.

Hausner's ratio is the ratio of bulk density (V0) to tapped density (Vf) of powder.

$$
\text { Hausner's ratio }=V 0 / V f
$$

Carr's index is indirect measure of bulk density, size, shape, surface area, moisture content and cohesiveness of material as all these factors affect compressibility.

$$
\text { Carr's index }=100 *\left(\frac{V_{0}-V_{t}}{V_{0}}\right)
$$

Where $\mathrm{V}_{0}$ is apparent bulk volume while $\mathrm{V}_{\mathrm{t}}$ is tapped volume of the powder. 
Table S1 Qualitative solubility of etodolac in various organic solvents

\begin{tabular}{|c|c|c|c|}
\hline S. No & Solvents & ICH class & Solubility $(\mathrm{mg} / \mathrm{ml})$ \\
\hline 1. & Methanol & II & 580 \\
\hline 2. & Ethanol & III & 320 \\
\hline 3. & Isopropyl alcohol & III & 490 \\
\hline 4. & Dichloromethane & II & 218 \\
\hline 5. & Chloroform & II & 493 \\
\hline 6. & Ethyl acetate & III & 420 \\
\hline 7. & Acetone & III & 473 \\
\hline 8. & Diethyl ether & III & 221 \\
\hline 9. & Acetonitrile & II & 360 \\
\hline 10. & Dimethyl sulphoxide & III & 600 \\
\hline 11. & Dimethyl formamide & II & 1300 \\
\hline 12. & Dioxane & II & 1275 \\
\hline
\end{tabular}

Table S2 Wells protocol for studying compressibility

\begin{tabular}{|l|c|c|c|}
\hline Sample amount & \multicolumn{3}{|c|}{$500 \mathrm{mg}+1 \%$ Magnesium stearate } \\
\hline Sample code & $\mathrm{A}$ & $\mathrm{B}$ & $\mathrm{C}$ \\
\hline Blending time & $5 \mathrm{~min}$ & $5 \mathrm{~min}$ & $30 \mathrm{~min}$ \\
\hline \multicolumn{4}{|c|}{ Compression using $13 \mathrm{~mm}$ die at $100 \mathrm{~kg} / \mathrm{cm}^{2}$ pressure } \\
\hline Dwell time & $2 \mathrm{~s}$ & $30 \mathrm{~s}$ & $2 \mathrm{~s}$ \\
\hline \multicolumn{4}{|c|}{ Equilibration in closed glass vials for 24 hour } \\
\hline
\end{tabular}

Table S3 Screening of the polymer; SA= Spherical agglomerates; IA= Irregular agglomerates

\begin{tabular}{|c|c|c|c|c|}
\hline \multirow{2}{*}{ Polymer } & \multicolumn{3}{|c|}{ Concentration (\% W/V) } \\
\cline { 3 - 5 } \multicolumn{2}{|c|}{} & $0.1 \%$ & $0.25 \%$ & $0.5 \%$ \\
\hline \multirow{3}{*}{ HPMC } & E3 & SA & IA & IA \\
\cline { 2 - 5 } & E5 & IA & IA & IA \\
\cline { 2 - 5 } & E15 & IA & IA & IA \\
\hline & L & IA & SA & IA \\
\hline
\end{tabular}




\begin{tabular}{|c|c|c|c|c|}
\hline \multirow{2}{*}{ HPC } & M & IA & IA & Paste \\
\cline { 2 - 5 } & $\mathrm{H}$ & Paste & Paste & Paste \\
\hline \multirow{3}{*}{ PVP } & $17 \mathrm{~K}$ & IA & IA & IA \\
\cline { 2 - 5 } & $25 \mathrm{~K}$ & Lumps & Lumps & Lumps \\
\cline { 2 - 5 } & $30 \mathrm{~K}$ & Paste & Paste & Paste \\
\hline
\end{tabular}

Table S4 Screening of the copolymers SA= Spherical agglomerates; IA= Irregular agglomerates

\begin{tabular}{|c|c|c|c|c|c|c|}
\hline $\mathbf{P}$ & olymers & $\begin{array}{c}\text { HPMC } \\
\text { E3 } \\
(0.1 \%)\end{array}$ & $\begin{array}{l}\text { HPC L } \\
(0.25 \%)\end{array}$ & $\begin{array}{c}\text { PVP 17k } \\
(0.1 \%)\end{array}$ & $\begin{array}{c}\text { HPC/E3 } \\
(0.1 \% / 0.1 \\
\%)\end{array}$ & $\begin{array}{l}\text { HPC/E3 } \\
(0.1 / 0.5 \%)\end{array}$ \\
\hline None & - & SA & SA & IA & SA & SA \\
\hline \multirow{3}{*}{ PEG 400} & $5 \%$ & $\mathrm{SA}$ & Clumps & Clumps & SA & SA \\
\hline & $10 \%$ & SA & Paste & Paste & IA & IA \\
\hline & $15 \%$ & IA & Paste & Paste & IA & IA \\
\hline \multirow{3}{*}{ PVA } & $0.1 \%$ & IA & Paste & Paste & IA & IA \\
\hline & $0.25 \%$ & IA & Paste & Paste & IA & IA \\
\hline & $0.5 \%$ & Paste & Paste & Paste & IA & IA \\
\hline \multirow{3}{*}{ Poloxamer } & $0.1 \%$ & $\mathrm{SA}$ & Paste & Paste & SA & SA \\
\hline & $0.25 \%$ & $\mathrm{SA}$ & Paste & Paste & SA & SA \\
\hline & $0.5 \%$ & IA & Paste & Paste & IA & IA \\
\hline
\end{tabular}

Table S5 DSC Onset, melting and endset points of formulations

\begin{tabular}{|l|c|c|c|c|}
\hline Code & Onset $\left({ }^{\mathbf{0}} \mathbf{C}\right)$ & Melting $\left({ }^{\mathbf{O}} \mathbf{C}\right)$ & Endset $\left({ }^{\mathbf{0}} \mathbf{C}\right)$ & Enthalpy (J/g) \\
\hline API & 149.30 & 150.73 & 154.90 & 123.39 \\
\hline E3 & 149.75 & 151.17 & 156.66 & 127.15 \\
\hline E3+PEG & 147.86 & 150.69 & 155.66 & 112.82 \\
\hline E3+HPC & 148.86 & 151.17 & 156.33 & 125.58 \\
\hline E3+POLOX & 148.66 & 150.78 & 156.65 & 119.05 \\
\hline E3+HPC+PEG & 148.26 & 150.67 & 156.20 & 115.70 \\
\hline E3+POLOX+HPC & 149.31 & 151.50 & 156.76 & 120.26 \\
\hline
\end{tabular}

Table S6 Flow evaluation of agglomerates $(n=3)$

\begin{tabular}{|l|l|l|l|l|l|}
\hline Code & $\begin{array}{l}\text { Angle of } \\
\text { Repose }\end{array}$ & Flow* & $\begin{array}{l}\text { Carr's } \\
\text { Index }\end{array}$ & $\begin{array}{l}\text { Hausner's } \\
\text { Ratio }\end{array}$ & Flow** \\
\hline E & 40 & Poor & 24 & 1.31 & Poor \\
\hline E3 & 25 & Excellent & 6.25 & 1.06 & Excellent \\
\hline
\end{tabular}




\begin{tabular}{|l|l|l|l|l|l|}
\hline E3+PEG & 28.5 & Excellent & 21 & 1.26 & Good \\
\hline E3+POLOX & 25 & Excellent & 12.28 & 1.14 & Excellent \\
\hline E3+HPC & 25 & Excellent & 17.6 & 1.2 & Good \\
\hline E3+HPC+PEG & 26 & Excellent & 12.5 & 1.14 & Excellent \\
\hline E3+HPC+POLOX & 26 & Excellent & 9.6 & 1.1 & Excellent \\
\hline
\end{tabular}

*Flow properties as concluded from angle of repose, $* *$ flow properties concluded from Carr's

Index and Hausner's ratio

Table S7 Hardness values and inference from compressibility studies $(n=3)$

\begin{tabular}{|l|c|c|c|c|c|}
\hline \multicolumn{1}{|c|}{ Code } & A & B & C & Hardness & Inference \\
\hline API & $2.9 \pm 0.35$ & $3.8 \pm 0.49$ & Capping & Capping & Elastic \\
\hline E3 & $4.8 \pm 0.84$ & $8.85 \pm 0.49$ & $3.6 \pm 0.56$ & $\mathrm{~B}>\mathrm{A}>\mathrm{C}$ & Plastic \\
\hline E3+PEG & $4.55 \pm 0.35$ & $6 \pm 0.63$ & $3.75 \pm 0.21$ & $\mathrm{~B}>\mathrm{A}>\mathrm{C}$ & Plastic \\
\hline E3+POLOX & $3.55 \pm 0.49$ & $5.9 \pm 0.42$ & $2.3 \pm 0.84$ & $\mathrm{~B}>\mathrm{A}>\mathrm{C}$ & Plastic \\
\hline E3+HPC (1:1) & $6.9 \pm 0.91$ & $8.35 \pm 0.63$ & $3 \pm 0.63$ & $\mathrm{~B}>\mathrm{A}>\mathrm{C}$ & Plastic \\
\hline E3+HPC (1:2) & $10.7 \pm 0.77$ & $11.7 \pm 0.56$ & $5.6 \pm 0.84$ & $\mathrm{~B}>\mathrm{A}>\mathrm{C}$ & Plastic \\
\hline E3+HPC+PEG & $4.75 \pm 0.63$ & $6.5 \pm 0.56$ & $3.6 \pm 0.56$ & $\mathrm{~B}>\mathrm{A}>\mathrm{C}$ & Plastic \\
\hline E3+HPC+POLOX & $5 \pm 0.77$ & $8.75 \pm 0.63$ & $3 \pm 0.21$ & $\mathrm{~B}>\mathrm{A}>\mathrm{C}$ & Plastic \\
\hline
\end{tabular}

Table S8 Drug release and dissolution efficiency at 15 minutes $(n=3)$

\begin{tabular}{|l|c|c|}
\hline \multicolumn{1}{|c|}{ Code } & $\mathbf{Q}_{\mathbf{1 5}}$ & DE $_{\mathbf{1 5}}$ \\
\hline API & 75 & 51 \\
\hline Control & 63 & 38.73 \\
\hline E3 & 43 & 27.62 \\
\hline E3+PEG & 49 & 32.83 \\
\hline E3+HPC-SA (1:1) & 68 & 43.15 \\
\hline E3+HPC-SA (2:1) & 70 & 42 \\
\hline E3+POLOX-SA & 80 & 50.84 \\
\hline E3+HPC+PEG-SA & 93 & 56.24 \\
\hline E3+HPC+POLOX & 69 & 47.75 \\
\hline
\end{tabular}


Figures

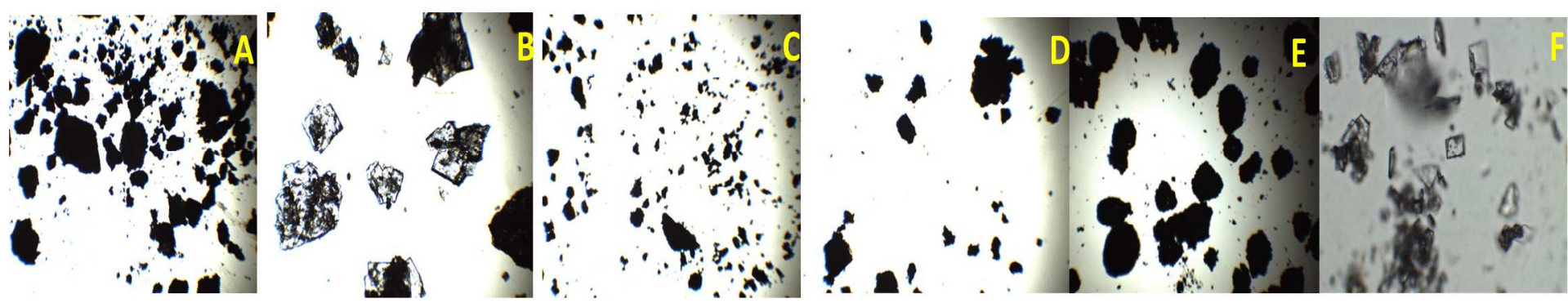

Figure S1 Agglomerates with different solvent system. A) Methanol/water/DCM; B) Acetonitrile/water/DCM C) Ethanol/water/DCM D) IPA/water/DCM E) Acetone/water/DCM and F) Acetone.
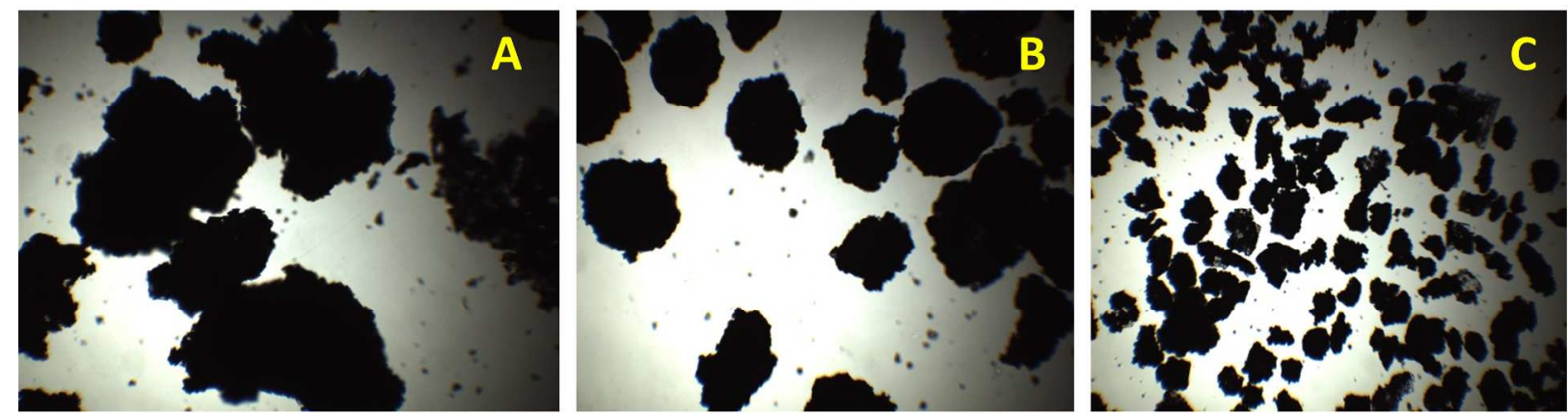

Figure S2 Effect of speed of rotation on the agglomerates in acetone-water-DCM A) 500 rpm;

B) $700 \mathrm{rpm}$; C) $900 \mathrm{rpm}$ 

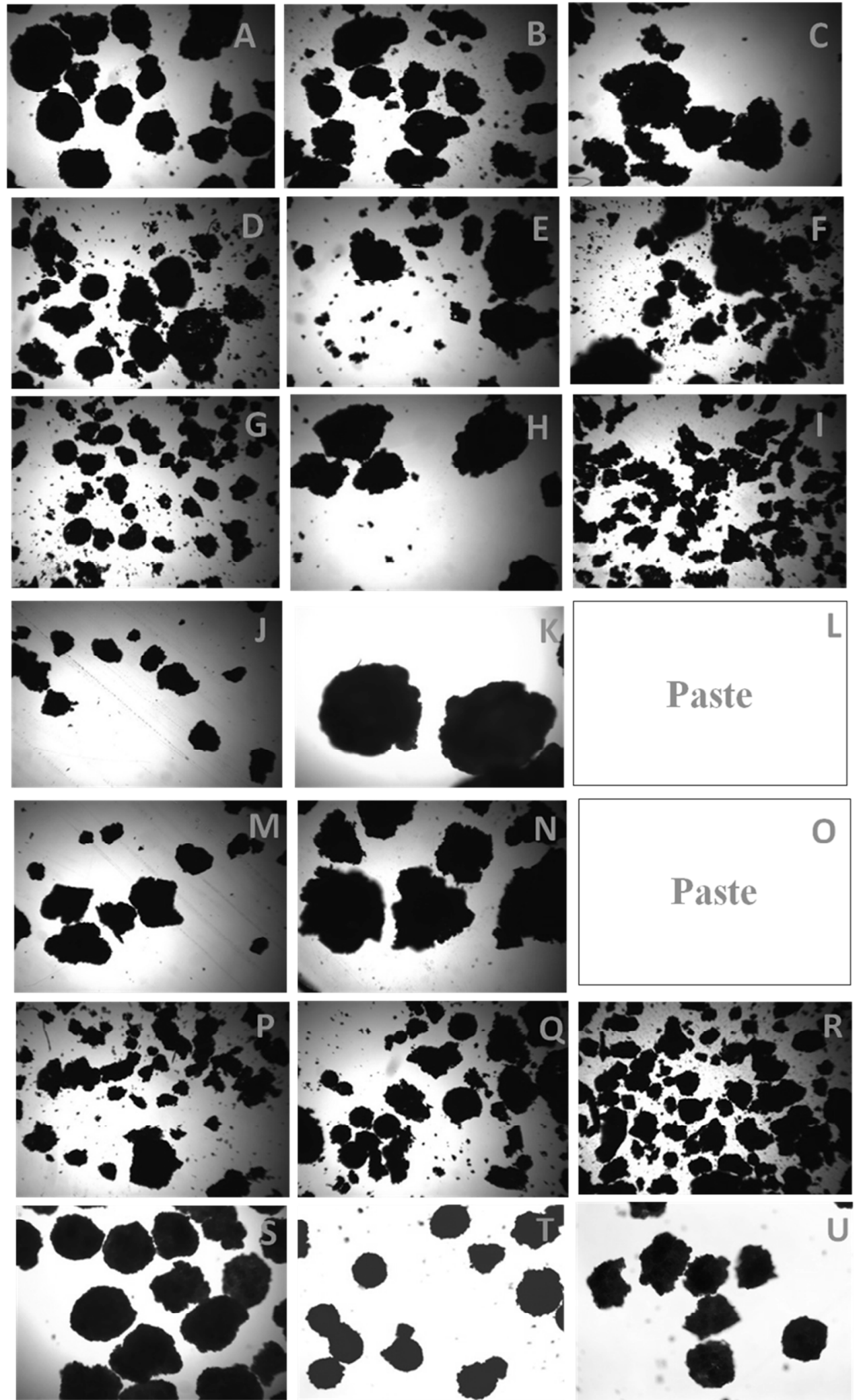

Figure S3 Agglomerates at $0.1,0.25$ and $0.5 \%$ polymer concentration of E3 (A, B, $\hat{\mathrm{C}}$ at $0.1 \%, 0.25 \%$ and $0.5 \%$ respectively); E5 (D, E, F at $0.1,0.25$ and $0.5 \%$ respectively); E15 (G, H, I at $0.1 \%, 0.25 \%$ and $0.5 \%$ respectively); HPC-L (J, K, L at $0.1,0.25$ and $0.5 \%$ respectively); HPC-M (M, N, O at $0.1,0.25$ and $0.5 \%$ respectively); PVP (P, $\mathrm{Q}, \mathrm{R}$ at $0.1,0.25$ and $0.5 \%$ respectively); $\mathrm{S}-\mathrm{E} 3+\mathrm{HPC}$ combination at $1: 1$ ratio $(0.1 \%$ each); $\mathrm{T}-\mathrm{E} 3+\mathrm{HPC}$ at $0.1 / 0.05 \%$ (2:1); U - E3+HPC 1:1 ratio (0.05\% each). 

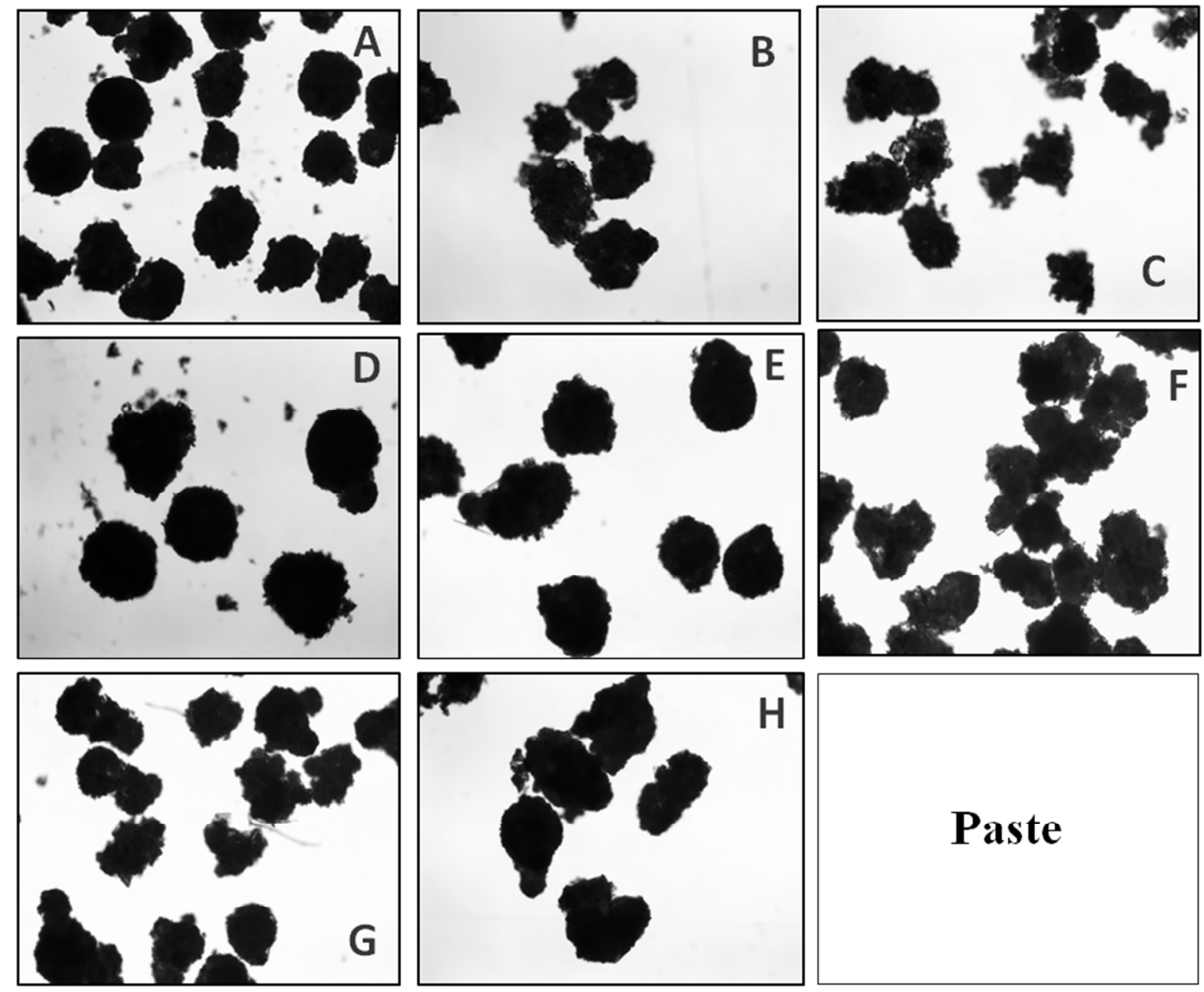

Figure S4 Microscopic images of E3 in combination with different copolymers; E3 with PEG (E3+PEG) $5 \%, 10 \%$ and $15 \%$ (A, B, C); E3 with Poloxamer (E3+POLOX) 0.1\%,0.25\%,0.5\% (D, E, F); E3 with PVA (E3+PVA)0.1 \% and $0.25 \%(\mathrm{G}, \mathrm{H})$. 

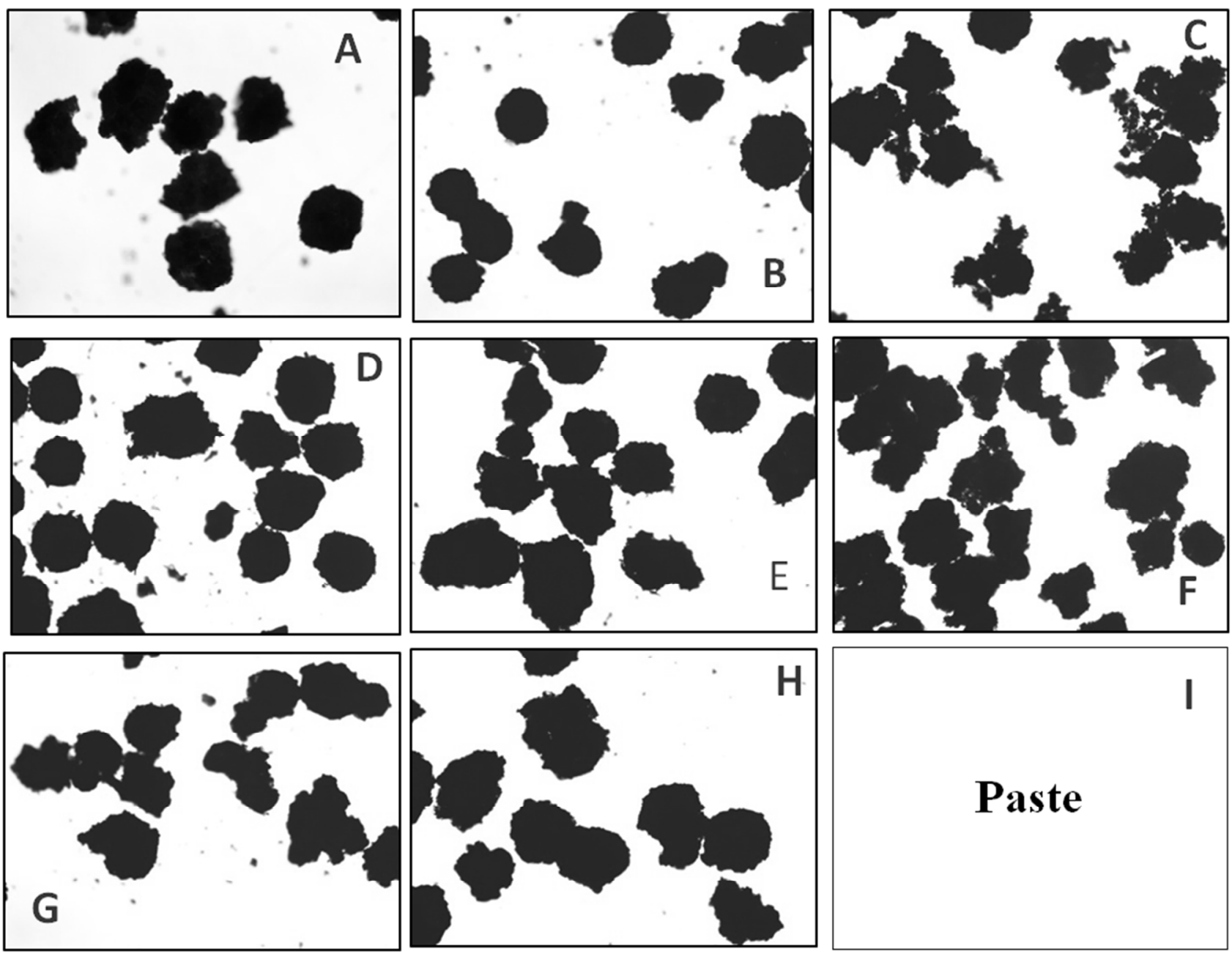

Figure S5 Microscopic images of E3+HPC (2:1) Combinations with PEG (E3+HPC+PEG)5 $\%, 10 \%$ and $15 \%$ (A, B, C); with poloxomer (E3+HPC+POLOX) $0.1 \%, 0.25 \%$ and $0.5 \%$ (D, E, F); with PVA (E3+HPC+PVA) $0.1 \%$ and $0.25 \%, 0.5 \%(\mathrm{G}, \mathrm{H}, \mathrm{I})$. 


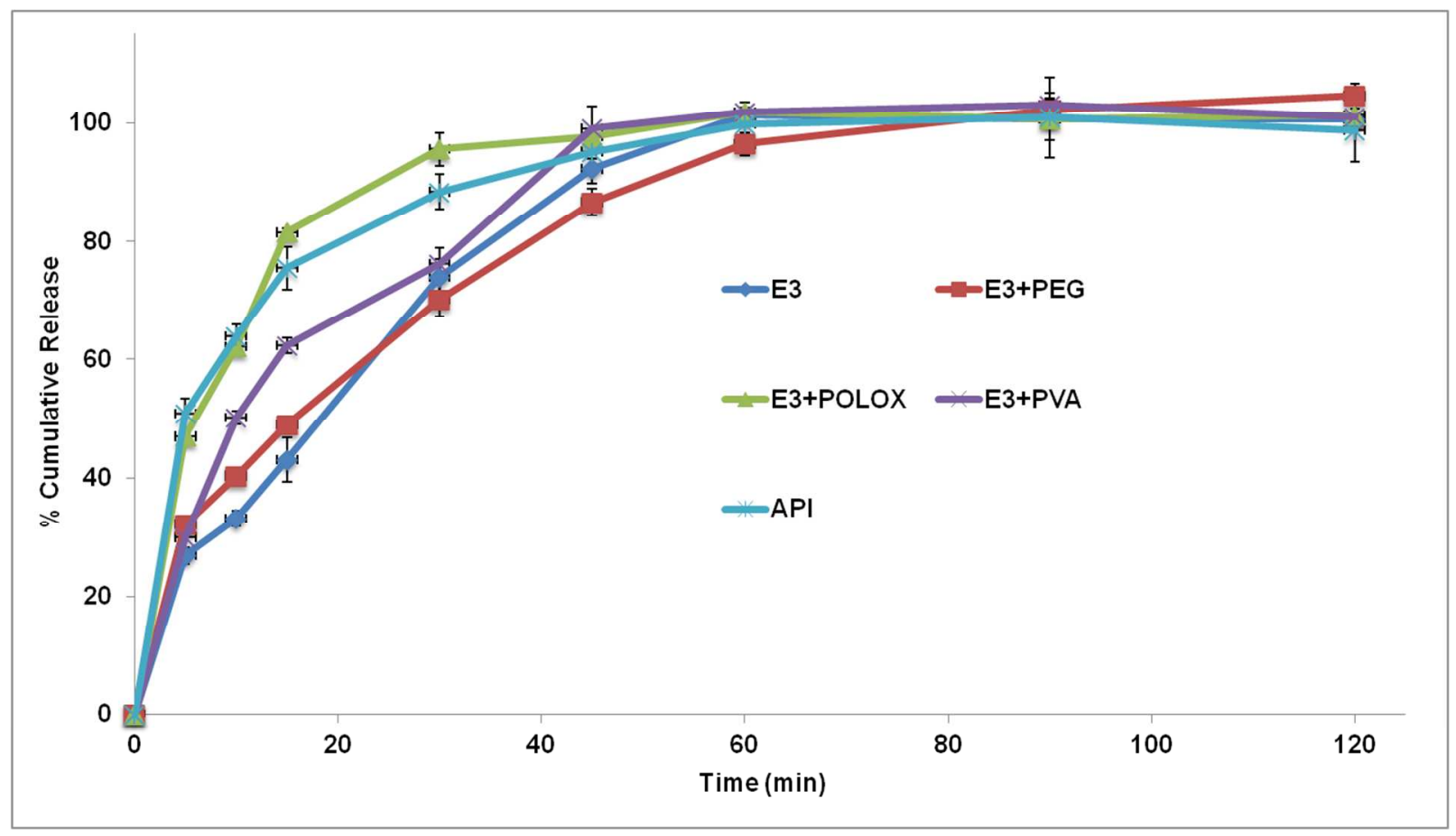

Figure S6 Dissolution profiles of E3 and copolymer combinations

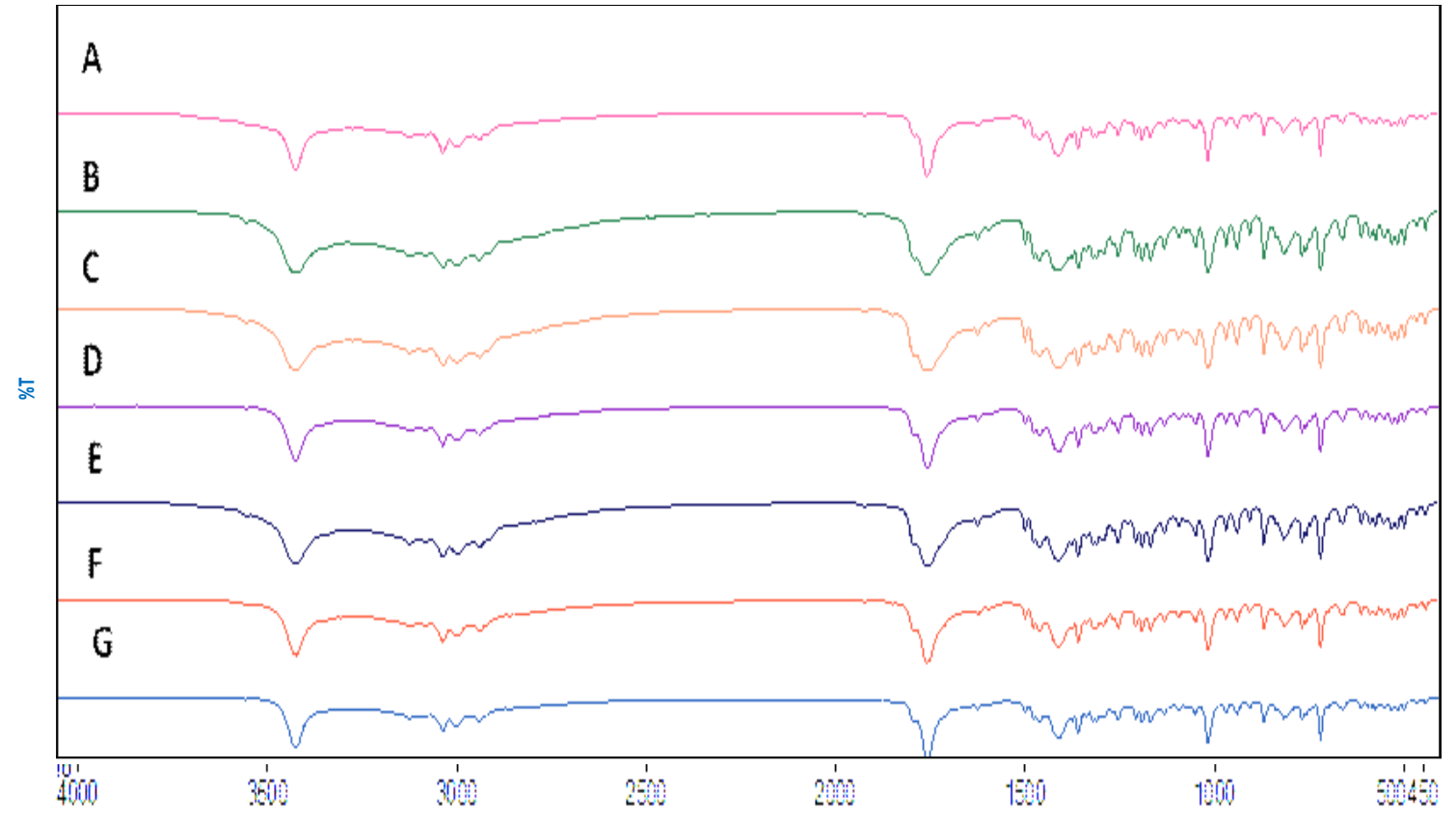

Figure S7 FT-IR spectra of plain drug with formulation A) Plain drug B) E3 C) E3 \%) E3+PEG D) E3+POLOX E) E3+HPC F) E3+HPC+PEG G) E3+HPC+POLOX 


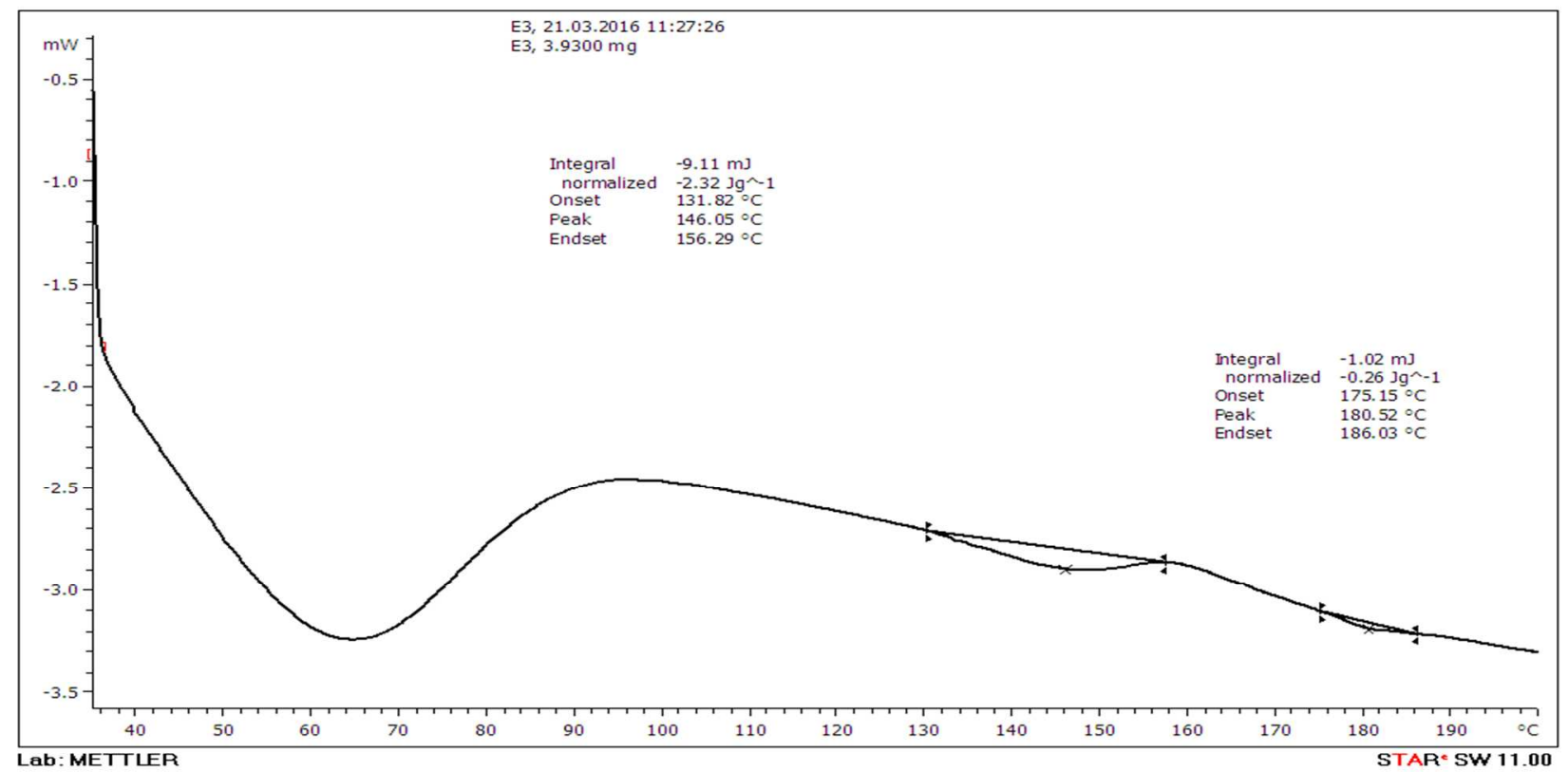

Figure S8 DSC thermogram of HPMC E3.

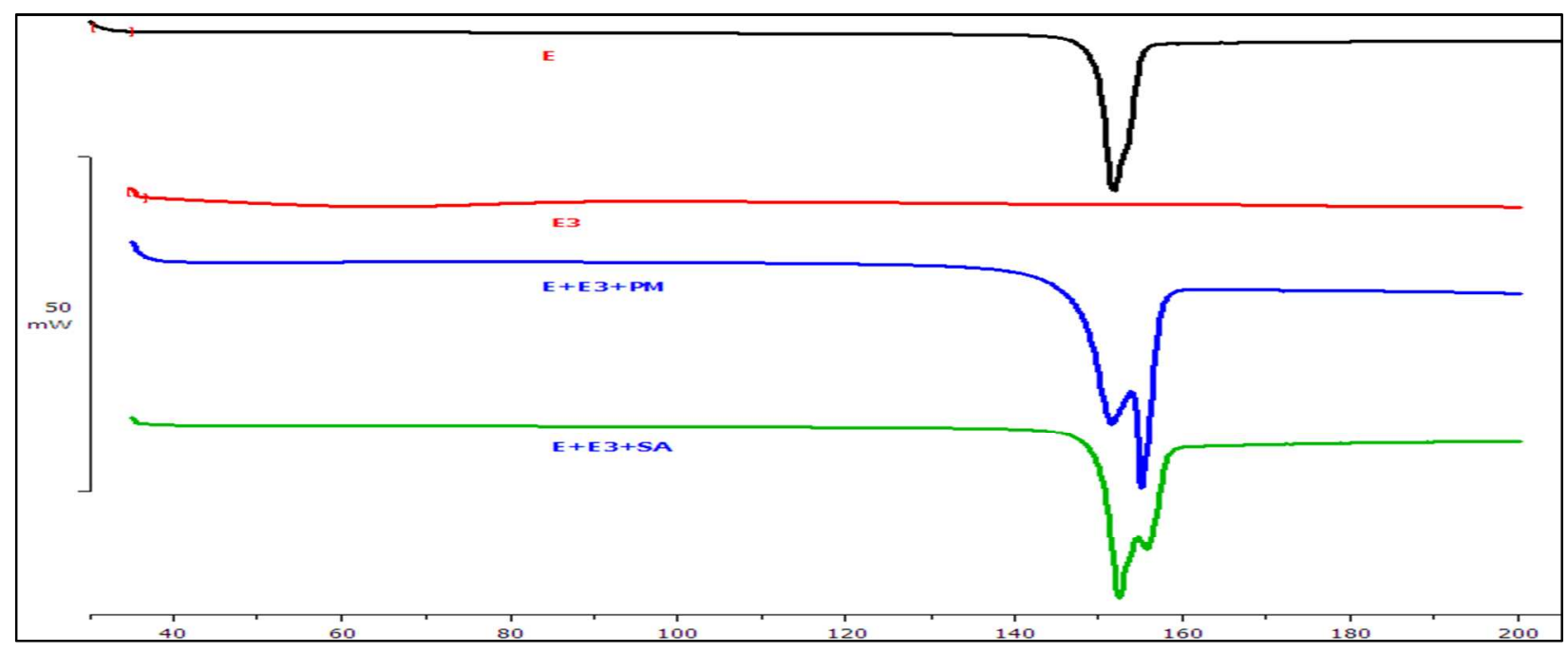

Figure S9 DSC thermograms of plain drug along with HPMC E3 formulations and physical mixture. 


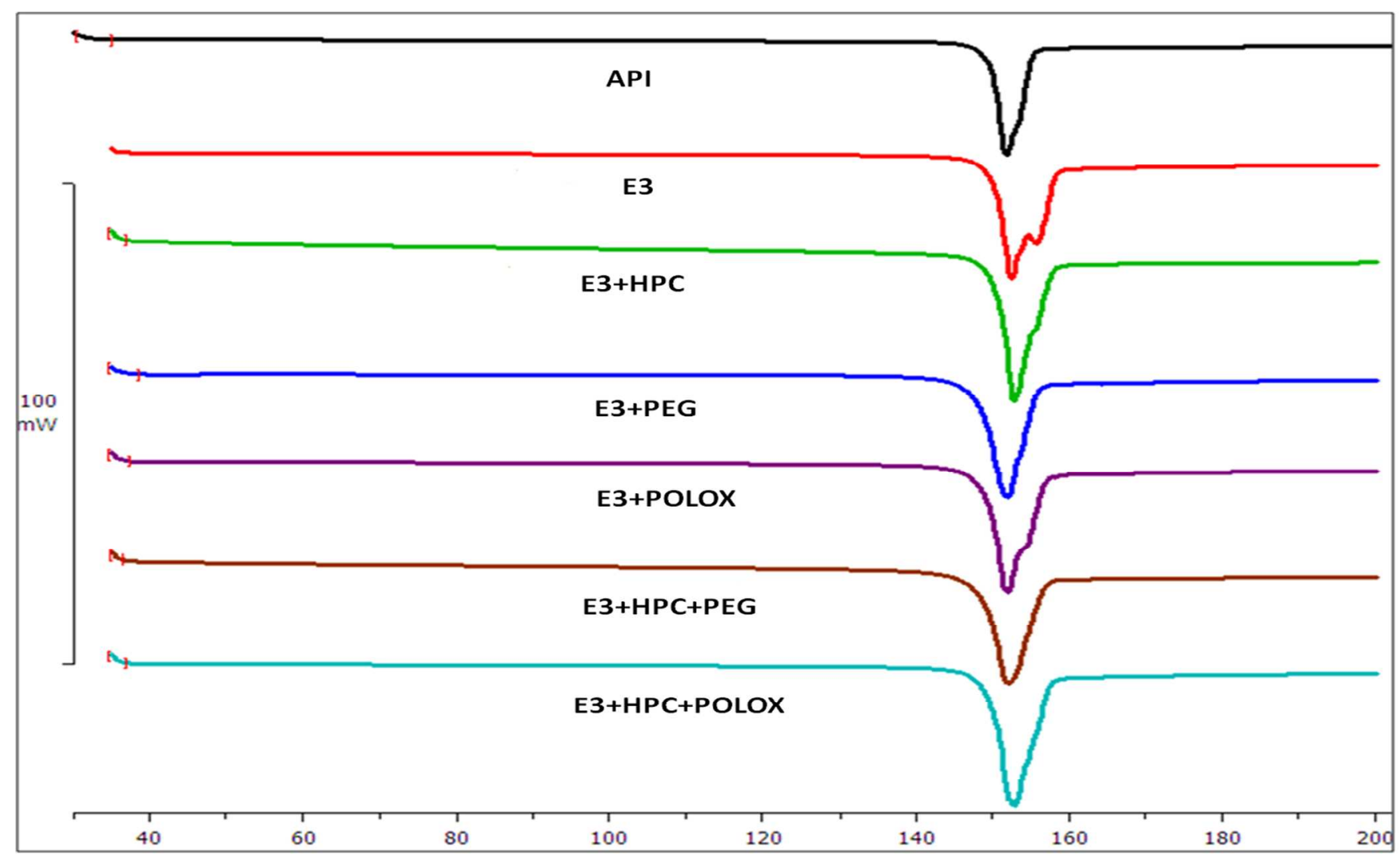

Figure S10 DSC thermograms of plain drug with formulations

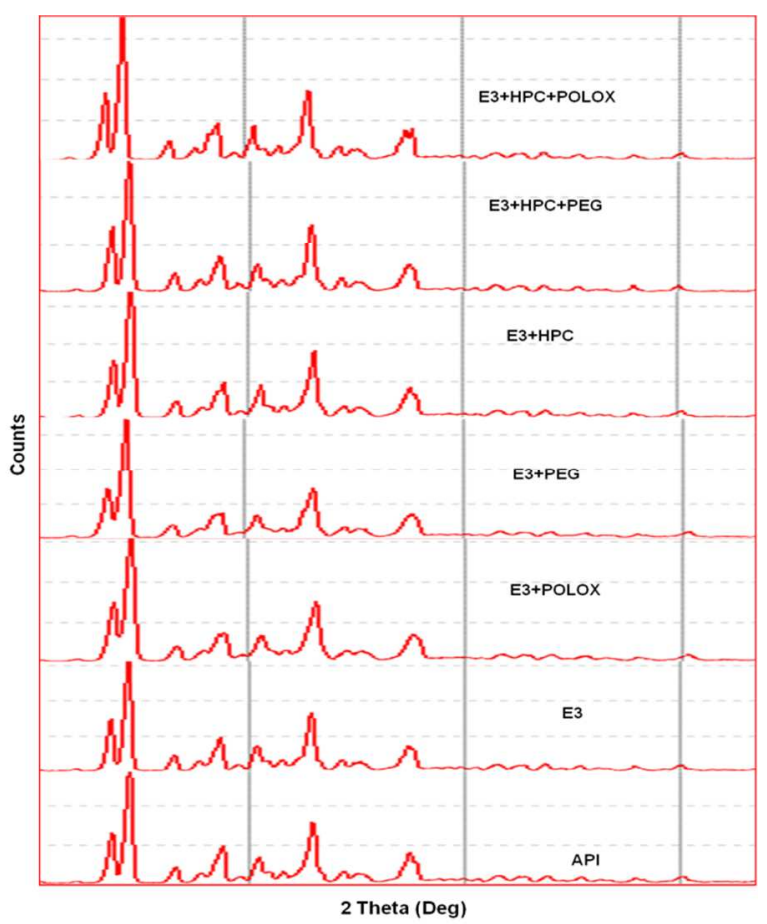

Figure S11 Overlay of powder diffractograms of formulations 


\section{References}

(1) Carr, R. L., Evaluating flow properties of solids. Chem. Eng. 1965, 72, 163-168.

(2) Shah, R. B.; Tawakkul, M. A.; Khan, M. A., Comparative evaluation of flow for pharmaceutical powders and granules. Aaps Pharmscitech 2008, 9, (1), 250-258. 